\section{RMD Open}

Rheumatic \&

Musculoskeletal Diseases

\title{
To move or not to move: the paradoxical effect of physical exercise in axial spondyloarthritis
}

\author{
Fabio Massimo Perrotta, ${ }^{1}$ Rik Lories, ${ }^{2}$ Ennio Lubrano (i) ${ }^{1}$
}

To cite: Perrotta FM, Lories R, Lubrano $\mathrm{E}$. To move or not to move: the paradoxical effect of physical exercise in axial spondyloarthritis. RMD Open 2021;7:e001480. doi:10.1136/ rmdopen-2020-001480

Received 14 0ctober 2020 Revised 16 January 2021 Accepted 18 January 2021

\section{Check for updates}

\section{(c) Author(s) (or their} employer(s)) 2021. Re-use permitted under CC BY-NC. No commercial re-use. See rights and permissions. Published by BMJ.

${ }^{1}$ Dipartimento di Medicina e Scienze della Salute "Vincenzo Tiberio", Universita degli Studi del Molise, Campobasso, Italy ${ }^{2}$ Skeletal Biology and Engineering Research Centre, Catholic University College Leuven Department of Development and Regeneration, Leuven, Belgium

Correspondence to Professor Ennio Lubrano; enniolubrano@hotmail.com

\section{ABSTRACT}

In the last years, new researches focused on the role of biomechanical stress and microdamage in the pathogenesis of inflammatory arthritis and, in particular, in axial spondyloarthritis (axSpA). Animal models showed how entheseal stress and physical exercise could contribute to the development of inflammation and new bone formation at entheseal and articular sites, by activating innate immune system and the release of cytokines. Furthermore, clues of the involvement of biomechanical stress in the development of axSpA are present in clinical experiences. However, rehabilitation and exercise programmes are the cornerstone of treatment for axSpA, reducing disease activity and improving spinal function and quality of life. The concept of mechanical stress as a contributor to disease development and progression represents, potentially, a conceptual challenge for this approach. The aim of this review is to discuss the current evidence on the intriguing contribution of the biomechanical stress to the pathogenesis of inflammation and new bone formation and to evaluate and reflect on the role of exercise in the treatment and in the management of the disease, considering both the beneficial effects and its possible paradoxical action.

\section{INTRODUCTION}

Spondyloarthritis $(\mathrm{SpA})$ represents a group of common chronic inflammatory diseases that share genetic, immunopathological, clinical, laboratory and radiological features. ${ }^{1}$ Within this cluster of diseases, axial SpA (axSpA) is characterised by inflammation in sacroiliac joints and the spine, with the possible presence of peripheral and extra-articular manifestations. ${ }^{2}$ Clinical signs such as inflammatory back pain, stiffness, swelling and loss of function are caused by enthesitis, osteitis, synovitis and new bone formation. ${ }^{1}$ The enthesis, an anatomical zone in which fibres of the tendons, ligaments and capsules insert into the bone through a fibrocartilaginous connection, has been hypothesised to be the primary disease localisation in axSpA. ${ }^{3}$ In this context, the entheseal inflammatory lesions and the subsequent new bone formation at

\section{Key messages}

What is already known about this subject?

- Spondyloarthritis represents a group of chronic inflammatory diseases in which biomechanical factors could be important in the pathogenesis and management.

- Animal models demonstrated a potential role for biomechanical stress in the pathogenesis of entheseal and joint inflammation, and, possibly, in the pathogenesis of new bone formation.

What does this study add?

- This review shows that exercise and physical activity are part of the human life and together with genetic and immunological factors, biomechanical stress and aberrant load might be related to the pathophysiology of axSpAaxial spondyloarthritis (axSpA).

How might this impact on clinical practice?

- In the management of axSpA, rehabilitation and even 'high' intensity activity are efficacious in the reduction of burden of disease, however, in the light of the recent evidence, a careful attention should be paid on the type of exercise.

these sites appear linked and can lead to functional impairment and disability, mainly in those patients who develop extensive syndesmophyte formation in the spine. Over the last few years, different preclinical and clinical studies, using MRI, demonstrated important roles for inflammation and biomechanical stress at entheseal sites in the pathogenesis of inflammatory changes and in the progression of bone damage in axSpA. ${ }^{4-8}$ Microdamage and subsequent reparative processes might be implicated in the development and progression of disease, together with the production of cytokines such as tumour necrosis factor (TNF) interleukin (IL)-22, IL-17 and IL-23 by the immune system. ${ }^{8}$

AxSpA is a chronic disease that could impact the patients for years, leading to functional impairment, reduced mobility and quality of life. Physical therapy and exercise 


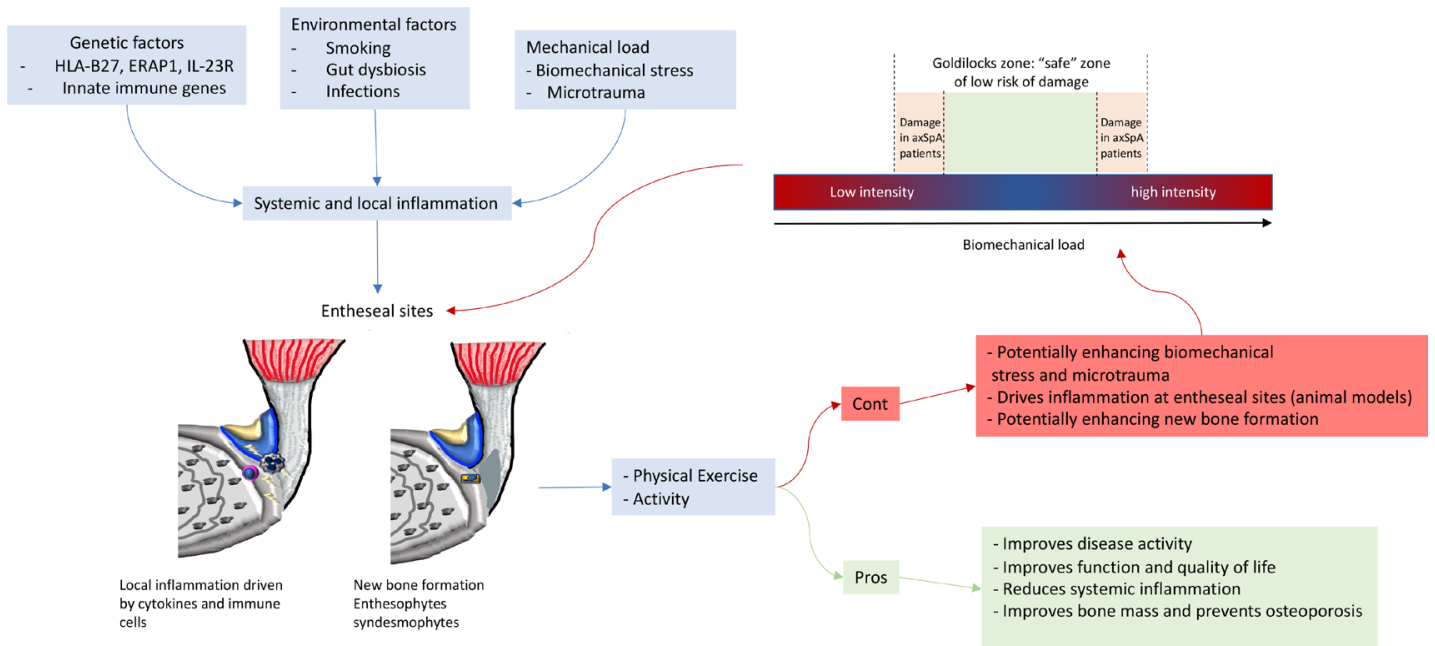

Figure 1 Proposed model of interaction of mechanical stress factors, genetics and environmental factors in the pathogenesis of enthesitis in SpA. The treatment approach with physical exercise and activity has showed to improve symptoms and sign of axSpA and to improve function and quality of life. However, potential harms could occur mainly for those patients with instability or heavy entheseal stress with a reduction of the Goldilocks zone. axSpA, axial spondyloarthritis; ERAP1, endoplasmic reticulum aminopeptidase-1; IL, interleukin.

are considered to be one of the cornerstones of the management of disease, especially for patients with severe radiographic involvement. ${ }^{9}$ Although rehabilitation and exercise programmes reduce disease activity and improve spinal function and quality of life, the concept of mechanical stress as a contributor to disease development and progression represents a conceptual challenge for this approach. Despite the observed benefits, specific or excessive exercise could potentially and paradoxically increase the extent of microdamage at entheseal sites, and, thus, worsen the disease outcomes. The paradox of the benefit and harm of activity in axSpA was conceptualised in the so called 'Goldilocks zone', an intriguing concept linked to the potential role of mechanical stress in the pathogenesis of SpA (figure 1). This narrative review aims to discuss current evidence on the intriguing contribution of the biomechanical stress to the pathogenesis of inflammation and new bone formation and to evaluate and reflect on the role of exercise in the treatment and in the management of the disease, considering both the beneficial effects and its possible paradoxical action. We searched PubMed and the Cochrane library for articles and reviews in the English language published between 1 January 2010 and 30 March 2020. Search terms were 'axial spondyloarthritis', 'ankylosing spondylitis', 'enthesitis', 'biomechanical stress', 'microdamage', 'new bone formation', 'pathogenesis', 'physical therapy', 'rehabilitation', 'exercise', and limited the search to preclinical studies, clinical studies, clinical trials, reviews and meta-analyses. Key abstracts were identified from the European League Against Rheumatism and American College of Rheumatology Annual Meetings.

We also used additional papers suggested by all coauthors, identified as important for the context of this review. The final reference list was generated on the basis of relevance to the concepts the authors aimed to highlight in the manuscript.

\section{PHYSIOPATHOLOGY OF AXSPA: GENETIC, OSTEO- IMMUNOLOGICAL FACTORS AND PATHWAYS CONTRIBUTE TO INFLAMMATION AND NEW BONE FORMATION Genetics}

The pathogenesis of axSpA is anchored in a complex interplay between genetic, environmental (infections, gut microbiota), immunological (monocytes, B and $\mathrm{T}$ lymphocytes, innate lymphoid cells, mesenchymal cells) and biomechanical factors. All of these factors can contribute to the hallmarks of the disease: inflammation and new bone formation. ${ }^{2}$

Evidence of genetic involvement in axSpA and in particular in ankylosing spondylitis (AS) is clinically striking and was first corroborated more than 45 years ago, by showing the strong association of HLA-B27 with AS. ${ }^{10}$ Although the precise role of HLA-B27 in the pathogenesis of axSpA still is not fully understood, it is notable that this genetic association remains the strongest link between variation in the major histocompatibility complex genes and any common disease.

Recently, Genome-Wide Association Studies have detected several alleles associated with axSpA. In one large study, $20 \%-44 \%$ of the genetic predisposition was linked to major histocompatibility complex variants (mainly HLA-B27, but also HLA-B40, HLA-B51, HLAB7, HLA-A2 and HLA-DPB1), and 7\%-8\% to non-HLA variants. The remaining proportion of genetic predisposition remains to be identified. In addition to HLA-B27, two further genetic loci have been particularly associated with axSpA and might be of functional relevance: polymorphisms in the endoplasmic reticulum aminopeptidase-1, and in the IL-23 receptor genes. ${ }^{212-14}$ 
HLA-B27 might be, per se, a risk factor for the development of axial new bone formation. ${ }^{15}$ Studies in rodent models showed that HLA-B27 transgenic rats develop inflammation, arthritis and spondylitis with joint erosions and with new bone proliferation in striking similarity to the observations in humans with AS. ${ }^{16}$ In addition, the presence of HLA-B27 was associated with factors that regulate bone remodelling: HLA-B27 positive subjects were reported to have substantially lower serum concentrations of proteins involved in the mechanism of inhibition of new bone formation. ${ }^{17}$ Furthermore, HLA-B27 positive patients were at higher risk to develop syndesmophytes when compared with HLA-B27 negative ones and, in general, having a more severe disease,.$^{15}$ Yet, it remains difficult to establish a direct link between HLA-B27 and disease progression, as the observations may be linked to the disease severity rather than a directly contributing mechanism. Misfolding of HLA-B27, a process in which the normal structural formation and assembly of proteins during protein synthesis is disturbed, was proposed as a mechanism linking HLA-B27 with the production of proinflammatory cytokines such us IL-23. ${ }^{18}$ In this model, the cellular response to misfolded proteins was responsible for proinflammatory cytokine secretion that drives inflammation and bone damage. Recently, Liu et al demonstrated, in animal models, that HLA-B27 misfolding activates different intracellular signalling cascades associated with cell stress and with potential roles in spinal ankylosis. ${ }^{19}$ Although of great interest, current translational studies such as the one mentioned above, face several challenges such as the use of endstage disease patient materials, impact of drugs used by the patients and the complex comparisons between cells derived from pathological specimens and those coming from a non-inflammatory environment.

\section{Osteo-immunological factors}

Several levels of evidence support the view that the IL-23/IL-17 pathway plays an important role in the development of axSpA: IL-23 is involved in the Th17-type immune responses, that are a major axis of immune activation present in axSpA patients. Moreover, numbers of Th17 cells are increased in AS patients, suggesting their role in the pathogenesis of the disease. IL-23 is essential for the proliferation and terminal differentiation of CD4+ Th17T cells, maintaining IL-17 production, and ultimately driving the cells pathogenicity. ${ }^{20-26}$ IL-23 likely also stimulates excessive IL-17 production by innate lymphoid cells. As further evidence, a cornerstone study by Sherlock $e t a l^{27}$ demonstrated that overexpression of IL-23 by hepatic injection of IL-23 minicircles drives enthesitis and aortitis in mice, in a process involving IL-22 and IL-17. IL-17 has also an important role in synovial inflammation and joint destruction in both animal models and clinical studies. IL-23 and IL-17 are involved in the inflammatory stress response to injury and may play a role in new bone formation at the cortical bone that resembles fracture repair. In this context, IL-23 and
IL-17 might produce an inflammatory microenvironment that encourages anabolic bone responses, linking inflammation with the process of bone formation. ${ }^{8}$ Other factors are also likely to be involved, including prostaglandin E2, a known inducer of osteoblast differentiation, and IL-22. ${ }^{28}$ Of note, a recent article demonstrated tissue resident populations of $\gamma \delta$ T-cells in enthesis that have transcript expression related to tissue repair and immunomodulation and identifies a subset (V81) able to produce IL-17 independently of IL-23 stimulation. This finding further underlines the complex pathogenesis of entheseal inflammation. ${ }^{29}$

Many questions on the role of IL-17 remain to be solved and the cytokine's effects may be strongly contextdependent. For instance, IL-17, despite also being an inhibitor of osteoblast function and able to trigger systemic bone loss, might also directly contribute to bone formation by promoting the local differentiation of mesenchymal precursor cells in a response-to-injury setting. ${ }^{29}$ The direct role of IL-17 in the pathogenesis of new bone formation is still a matter of debate: while some studies demonstrated that bone loss induced by ovariectomy was increased in mice deficient for IL-17 receptor A (IL-17RA), and thus suggesting a protective and anabolic role of $\mathrm{IL}-17{ }^{30}$ other evidence showed that IL-17A inhibits protein expression of markers of late osteoblast differentiation, such as osteocalcin, and of early osteocyte differentiation, with a negative effect on osteoblast function and differentiation. ${ }^{31}$

\section{Pathways of bone metabolism}

In axSpA, there are several levels of evidence that inflammation is linked to new bone formation. However, the precise mechanism by which new bone formation is activated and its relationship with proinflammatory cytokines remains to be clearly elucidated. The first anabolic pathway linked with the new bone formation process were bone morphogenetic proteins. These growth factors, members of the transforming growth factor-beta superfamily were first identified as morphogens that can induce bone formation in vivo. ${ }^{32}$ Studies in a dedicated model of ankylosing enthesitis suggested a key role in the new bone formation process in SpA and were corroborated by a targeted intervention in mice and their elevated levels in entheseal tissue from SpA patients. ${ }^{33}$ Subsequently, the role of the Wnt/ $\beta$-catenin pathway and its inhibitors, sclerostin and Dikkopf proteins, has been evaluated in axSpA pathogenesis to identify a possible link with bone formation. ${ }^{34} 35$ Diarra $e t a l^{36}$ demonstrated that activation of Wnt signalling determines the difference between a destructive rheumatoid arthritis-like phenotype of the human TNFtransgenic mouse model as opposed to a SpA-like phenotype when a Wnt antagonist is blocked. In the canonical pathway, Wnt proteins bind to a receptor/coreceptor complex on the plasma membrane, which consists of LRP5/ 6 and Frizzled proteins. The engagement of this receptor complex by Wnt proteins leads to cytoplasmic 
accumulation of $\beta$-catenin stimulating the molecule's translocation into the nucleus where it is involved in the transcription of genes responsible for osteoblast differentiation and bone formation. ${ }^{37}$ Activation of $\mathrm{Wnt} / \beta$ -catenin may promote bone formation in AS and other SpA. ${ }^{38}$ Similarly, a reduction in serum levels of sclerostin and Dikkopf1, two inhibitors Wnt/ $\beta$-catenin pathway, has been suggested in AS patients. ${ }^{39}$

Bone morphogenic proteins and the Receptor Activator of Nculear Factor-kB and its ligand (RANK/RANKL) system were also evaluated as possible biomarkers of bone remodelling. ${ }^{40}{ }^{41}$ However, further investigations are needed to validate all these molecules as true biomarkers of the ankylosing process because of the high variability in the methods and the lack of strong evidence as demonstrated in a recent meta-analysis. ${ }^{42}$ The major challenge with these biomarkers is their effective validation in large prospective cohorts and their independency of the disease or treatment associated factors, a challenge that up till now has not been met.

\section{PATHOPHYSIOLOGY OF AXSPA: THE ROLE OF MECHANICAL STRESS AT TENDON/ENTHESEAL SITES IN THE DEVELOPMENT OF INFLAMMATION AND NEW BONE FORMATION}

The entheseal organ concept defines a complex structure composed by different tissues and cell types that play an important role in the dissipation of mechanical stress. Tenocytes, chondrocytes, resident macrophages, gamma-delta $\mathrm{T}$ cells and innate lymphoid cells within the enthesis and tendon are involved in the mechanisms of tissue repair after an injury and, more in general, in the response to microdamage. A recent review by Gracey $e t a t^{43}$ extensively discussed the physiopathology and the possible links, that may occur, between repeated microdamage at tendon and entheseal sites and the development and progression of inflammation and bone damage in inflammatory arthritis, particularly in SpA. Figure 2 summarises the possible interaction between mechanical stress and osteo-immunological factors at entheseal sites. Mechanical stress, microdamage and loss of mechano-transduction signalling in entheseal tenocytes may lead to cell death and release of inflammatory cytokines. These molecules can propagate cell death in adjacent tenocytes and can promote the recruitment of proinflammatory cells, which perpetuates the inflammatory cycle by releasing proinflammatory molecules such as TNF, IL-6, IL-17 and prostaglandin E2. ${ }^{43}$ Aberrant or excessive repair responses in axSpA might be the initial phenomenon that leads to new bone formation. This intriguing aspect is difficult to investigate in human; however, different mouse models of arthritis were used to assess the possible role of biomechanical stress.

Different studies were performed in the mouse model of collagen-induced arthritis (CIA) and in the endogenous TNF overexpression mouse model (TNFdARE mice). Methods to investigate the role of biomechanical stress covered the spectrum of unloading to excessive loading using tail suspension and running as opposites. In a pioneer study, hind paw unloading prevented the onset of CIA without impairing the induction of anticollagen specific antibodies. Moreover, histological assessment of hind paws showed significant differences in total inflammation scores between unloaded and control mice, most strikingly around the achilles tendon and the ankle joint. On the other hand, the same authors showed that the excess mechanical load, by voluntary running accelerates the onset of arthritis induced by passive transfer of anticollagen specific antibodies. ${ }^{44-46}$ In another study, the same group showed that, in CIA mice, voluntary running contributed to the persistence of arthritis compared with

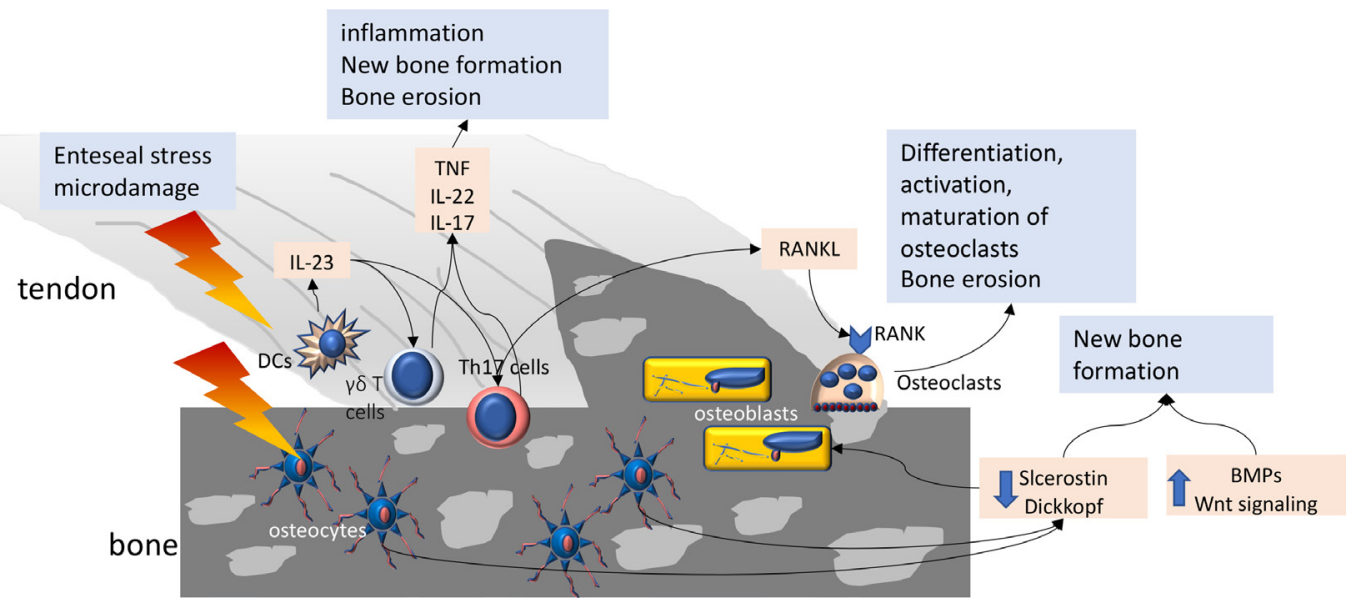

Figure 2 Mechanical stress and biologic actors possibly involved in on bone remodelling in SpA. Mechanical stress and microdamage could enhance IL-23 production which stimulates IL-17 production by $\gamma \delta$ T cells and Th17 cells. Proinflammatory cytokines contribute to inflammation and bone damage. RANKL may also be produced by $\mathrm{T}$ cells and contributes to maturation, activation and differentiation of osteoclasts. Finally, mechanical stress may reduce the production of sclerostin and Dickkopf proteins by osteocyte. Together with the increase of Wnt signalling and BMPs activity, new bone formation is enhanced. IL, interleukin; RANKL, Receptor Activator of Nculear Factor-kBBone Morphogenic protein; TNF, tumour necrosis factor. 
observations in control mice. Mechano-stimulation of mesenchymal cells induces the production of CXCL1 and CCL2 and recruitment of classical monocytes that may differentiate into bone-resorbing osteoclasts. Furthermore, the data provided in this study corroborated the hypothesis that mechanical strain is an essential checkpoint for the translation of systemic immune activation into site-specific joint inflammation. These data suggested a clear link, in mouse models, between biomechanical strain at entheseal sites and the developed tissue-specific inflammation. ${ }^{46}$

Moreover, further evidence linked the presence of biomechanical stress with the possible development of new bone formation (eg, enthesophytes at entheseal site). In the study by Jacques $e t a l,{ }^{6}$ osteophyte formation occurred after resolution of the inflammatory phase in a mouse model of CIA induced in DBA/1 mice. When clinical arthritis was present, half of the group of mice were tail suspended for 28 days to prevent mechanical loading on hind paws, and half were kept in normal cages. In tail suspended mice, osteophytes were significantly smaller compared with control mice, as shown by histology and micro-CT scan. Of note, osteophyte size correlated with the severity of inflammation. No new bone formation was observed in mice with minimal signs of inflammation and osteophytes when tail suspended. ${ }^{6}$

At present, clinical trials with anti-TNF and anti-IL-17A drugs showed a potential effect on radiographic progression, at least in a long-term perspective or in the context of early therapy initiation. ${ }^{47-49}$ Furthermore, the reduction of inflammation at sacroiliac joints and vertebral corners, and the reduction of fatty lesions indicates the opportunity to obtain a better radiographic outcome, in patients treated with these drugs, by reducing the inflammatory lesions that trigger new bone formation. ${ }^{47-49}$ However, besides pharmacological strategies, little is known about the link between repetitive entheseal stress and the progression of axSpA and, to our knowledge, no data are available on the possibility to reduce radiographic progression or delay the disease onset, by reducing or altering physical stress at entheseal sites in humans.

More recently, the observation that long-term inhibition of inflammation by cytokine blockage does appear to have an effect on structural disease progression in axSpA led to the hypothesis that-in the spine-loss of stability and mechanical resistance towards loading in affected sites associated with inflammation-induced bone loss, represent a strong trigger for the new bone formation process. ${ }^{50}$ In this perspective, the formation of syndesmophytes represents an effort of the body to increase stability by new bone formation, distant from the site of inflammation. Whereas this effort may theoretically be successful in changing the mechanical loading of the spine, the resulting disability by ankylosis clearly indicates that the intrinsic repair process is ill-oriented.
THE ROLE OF PHYSICAL EXERCISE IN AXSPA: TO MOVE OR NOT TO MOVE?

As stated above, biomechanical stress and entheseal microdamage could be strongly linked to the pathogenesis of inflammation and bone damage in axSpA. Moving these aspects from preclinical to clinical settings may potentially have some implications on the management of SpA patients. In fact, physical therapy and exercise have been used for years for the treatment of axSpA patients with the aim to reduce disability, enhance tendon elasticity and improve the range of motion and muscle tone and trophism. When taking into account the perspective of mobility and of spinal stability by muscle control, it is clear why such an approach should be beneficial if properly performed.

Indeed, physical therapy interventions for axSpA have shown to be an important part of the disease management, with a tendency to be more effective when done as a supervised outpatient group. ${ }^{51-55}$ The cornerstone of physical therapy in axSpA is regular exercise ${ }^{9}$ shown to reduce disease activity, pain and stiffness and improve physical functioning, chest expansion, spinal mobility and cardiorespiratory performance in axSpA patients. It also has the potential to reduce depressive symptoms. ${ }^{567}$ Evidence of its efficacy and effectiveness is strong, mainly when associated with pharmacological treatment. The trials consistently demonstrated the beneficial effect of a combination of anti-TNF therapy and an AS-specific exercise programmes, compared with either anti-TNF treatment or exercise alone, for both self-reported measures (such as function and disease activity) and objective measures, such as the Bath Ankylosing Spondylitis Metrology Index. ${ }^{56-58}$ Furthermore, ASAS/EULAR recommendations clearly identified exercise as a key component of axSpA management. ${ }^{59}$

It is not known whether the beneficial effects of exercise occur at a systemic (eg, anti-inflammatory) or local (eg, enthesis) level. In healthy adults, and those with a number of chronic diseases, exercise can produce systemic anti-inflammatory effects. However, at entheseal sites, anatomical and pathological changes in human axSpA after exercise are less known and remain an important topic for further research.

\section{Exercise and entheseal stress: what's wrong?}

It may be that exercise can have either proinflammatory or anti-inflammatory consequences for individuals with inflammatory arthritis, mainly due to the type of exercise and the local or systemic disease activity. Studies in healthy volunteers showed that physical activity is associated with changes in entheses on ultrasonographic evaluation, and, in psoriatic arthritis, patients who reported avoiding activities had significantly lower inflammatory lesions on imaging. ${ }^{60}$ Moreover, some studies on patients with axSpA have shown that blue collar workers have a greater disease progression than white collar workers with less physically demanding jobs. ${ }^{61} 62$ These data may suggest an influence of more physically demanding jobs 
on the onset and progression of axSpA disease, enhancing the role of biomechanical stress on the pathophysiology and progression of disease; however, confounding factors must be considered, such as socioeconomic status and smoking. Furthermore, male sex is clearly associated with radiographic damage and it should be considered that, in our society, men tend to have more load bearing jobs than women. This, together with biological, immunological and genetic factors may explain the gender differences in axSpA patients. ${ }^{62}$

Finally, there is the evidence, in healthy people, that physical activity decreases sclerostin levels with potential enhancement of new bone formation, although there are some studies showing an increase of sclerostin levels after intense physical activity. ${ }^{63}$

Another important aspect to be considered is the unloading of the enthesis. Sedentary lifestyle may have an impact on the complex biomechanical events at entheseal sites. Reduced loading could result in significant structural consequences at several hierarchical scales, with an increased risk of mechanical failure, as demonstrated in mouse models. ${ }^{64}$ Finally, in keeping with this finding, some reports showed that many patients with axSpA reported injuries or trauma as a potential trigger for their disease onset. ${ }^{6265}$

The paradox of the benefit and harm of activity in axSpA was conceptualised in the so called 'Goldilocks zone'. Very low activity could be detrimental owing to loss of strength and joint instability, whereas very high activity could damage the enthesis by repeating trauma. Goldilocks zone is considered a 'safe' zone of low risk of damage. In axSpA, a reduction of the Goldilocks zone was suggested compared with healthy individuals, with an associated higher risk of developing entheseal, tendon and bone damage, in association with genetic, immunological and microbiological factors. ${ }^{61}$ For example, young military recruits who experienced intense physical training with very little conditioning often develop exercise-related injuries, and the presence of bone marrow oedema at sacroiliac joints in different subjects without AxSpA that experienced intense physical activities has been demonstrated. ${ }^{61}$ Overall, the data could indicate that the relation between physical stress and onset or evolution of axSpA is influenced by a myriad of factors including baseline physical condition, lifestyle habits and type of exposure to biomechanical factors. ${ }^{61}$

On the other hand, a recent randomised controlled trial showed that 'high' intensity activity is efficacious not only in the reduction of burden of disease but even in the improvement of disease activity in patients with axSpA. ${ }^{66} 67$ This debunks concerns that high intensity exercise by default exacerbates disease activity in patients with axSpA. These results could be an example, in clinical real-life situation, of the 'paradoxical' effect of exercise as beneficial in axSpA. In fact, if exercise is effective in the reduction of disease activity and in the improvement of function compared with no exercise all the preclinical evidence (discussed above) and the above-mentioned studies raised some practical questions. Exercise plans likely will require a stronger personalised approach that takes into account the level of systemic and local fitness of the individual.

Finally, in axSpA and, to our knowledge, no studies were performed on the impact of exercise and physical therapy on the long-term radiographic progression of the disease. In other words, it could be important to demonstrate a possible 'disease modifying' role of exercise and physical therapy (with or without pharmacological treatment) in long-term outcomes in axSpA patients. Prospective randomised studies on this topic could be difficult to perform, but evidence may be found in existing cohorts and using advanced analysis methods.

Another interesting recent finding is linked to the well-known risk of low bone mineral density (BMD) in patients with axSpA, a feature associated with the abovementioned concept that lack of spinal stability can be a trigger for ankylosis. Kim $e t a b^{68}$ in their work found that low BMD was independently associated with the development of new syndesmophytes in young axSpA patients over a period of 2 years. The presence of low BMD in axSpA is considered to be a result of altered bone remodelling caused by persistent inflammation. While inflammatory mediators may act on the differentiation and activity of osteoclasts and osteoblasts mainly at trabecular level, a compensatory response of the cortical bone might be related to new bone formation. Anti-inflammatory biologic treatment reduces inflammation and allows the bone metabolism to normalise, thereby taking away the need for a compensatory anabolic response that leads to new bone formation in the cortical bone of the spine. ${ }^{68}$ In this light, physical exercise could have a possible positive effect on bone metabolism due to its efficacy in the improvement of BMD even in young men $^{69}$ and by resulting in increased core stability.

\section{CONCLUSIONS}

In conclusion, data coming from preclinical studies underlined the role of biomechanical stress and microdamage at entheseal sites in the development of tissue specific inflammation and subsequent new bone formation, in which, complex interaction between proinflammatory cytokines and pathways with growth factors and tissue-resident cells likely play critical roles.

Exercise and physical activity are part of the human life, however, together with genetic and immunological factors, biomechanical stress and aberrant load might be related to the pathophysiology of axSpA. How these activities could impact on disease progression is still poorly understood and further studies are needed on this intriguing topic, so a definitive and comprehensive answer to the question 'to move or not to move?' cannot be given yet. However, exercise and physical therapy proved their efficacy and effectiveness in the treatment of axSpA, by acting at different levels, not only in the reduction of clinical disease activity and symptoms but even by improving 
bone loss and general health. Furthermore, the nature of the movement (tension, compression, stretching and torsion), the repetition of the movement, moving rapidly from inactivity to activity and the anatomical site could be important. On this topic, further studies are needed to provide evidence on the possible role of the different type of exercises and physical therapy, mainly to give a better care in patients with axSpA. In fact, understanding what type of physical activity it may be associated, in the long-term period, with an improvement or worsening of the outcome in terms of new bone formation and radiographic progression towards ankylosis, can help in the management and education of patients.

Contributors All listed authors equally contributed to the planning, conduct, collection of data and in the writing of the work.

Funding The authors have not declared a specific grant for this research from any funding agency in the public, commercial or not-for-profit sectors.

Competing interests None declared.

Patient consent for publication Not required.

Provenance and peer review Not commissioned; externally peer reviewed.

Data availability statement № data were used for this review.

Open access This is an open access article distributed in accordance with the Creative Commons Attribution Non Commercial (CC BY-NC 4.0) license, which permits others to distribute, remix, adapt, build upon this work non-commercially, and license their derivative works on different terms, provided the original work is properly cited, appropriate credit is given, any changes made indicated, and the use is non-commercial. See: http://creativecommons.org/licenses/by-nc/4.0/.

ORCID iD

Ennio Lubrano http://orcid.org/0000-0001-6189-5328

\section{REFERENCES}

1 Garg N, van den Bosch F, Deodhar A. The concept of spondyloarthritis: where are we now? Best Pract Res Clin Rheumatol 2014;28:663-72.

2 Sieper J, Poddubnyy D. Axial spondyloarthritis. Lancet 2017;390:73-84

3 Benjamin M, McGonagle D. The anatomical basis for disease localisation in seronegative spondyloarthropathy at entheses and related sites. J Anat 2001;199:503-26.

4 Neerinckx B, Lories RJ. Structural disease progression in axial spondyloarthritis: still a cause for concern? Curr Rheumatol Rep 2017;19:14.

5 Poddubnyy D, Sieper J. Mechanism of new bone formation in axial spondyloarthritis. Curr Rheumatol Rep 2017;19:55.

6 Jacques P, Lambrecht S, Verheugen E, et al. Proof of concept: enthesitis and new bone formation in spondyloarthritis are driven by mechanical strain and stromal cells. Ann Rheum Dis 2014;73:437-45.

7 Dougados M, Sepriano A, Molto A, et al. Sacroiliac radiographic progression in recent onset axial spondyloarthritis: the 5-year data of the DESIR cohort. Ann Rheum Dis 2017;76:1823-8.

8 Gravallese EM, Schett G. Effects of the IL-23-IL-17 pathway on bone in spondyloarthritis. Nat Rev Rheumatol 2018:14:631-40.

9 Perrotta FM, Musto A, Lubrano E. New insights in physical therapy and rehabilitation in axial spondyloarthritis: a review. Rheumatol Ther 2019;6:479-86.

10 Brewerton DA, Hart FD, Nicholls A, et al. Ankylosing spondylitis and HL-A 27. Lancet 1973;1:904-7.

11 Schlosstein L, Terasaki PI, Bluestone R, et al. High association of an HL-A antigen, W27, with ankylosing spondylitis. N Engl J Med 1973;288:704-6.

12 Wellcome Trust Case Control Consortium, Australo-Anglo-American Spondylitis Consortium (TASC), Burton PR, et al. Association scan of 14,500 nonsynonymous SNPs in four diseases identifies autoimmunity variants. Nat Genet 2007;39:1329-37.

13 International Genetics of Ankylosing Spondylitis Consortium (IGAS), Cortes A, Hadler J, et al. Identification of multiple risk variants for ankylosing spondylitis through high-density genotyping of immunerelated loci. Nat Genet 2013:45:730-8.

14 Evans DM, Spencer CCA, Pointon JJ, et al. Interaction between ERAP1 and HLA-B27 in ankylosing spondylitis implicates peptide handling in the mechanism for HLA-B27 in disease susceptibility. Nat Genet 2011;43:761-7.

15 Coates LC, Baraliakos X, Blanco FJ, et al. The phenotype of axial spondyloarthritis: is it dependent on HLA-B27 status? Arthritis Care Res 2020. doi:10.1002/acr.24174. [Epub ahead of print: 26 Feb 2020]

16 van Duivenvoorde LM, Dorris ML, Satumtira N, et al. Relationship between inflammation, bone destruction, and osteoproliferation in the HLA-B27/human $\beta 2$-microglobulin-transgenic rat model of spondylarthritis. Arthritis Rheum 2012;64:3210-9.

17 Aschermann S, Englbrecht M, Bergua A, et al. Presence of HLA-B27 is associated with changes of serum levels of mediators of the Wnt and hedgehog pathway. Joint Bone Spine 2016;83:43-6.

18 Colbert RA, DeLay ML, Layh-Schmitt G, et al. HLA-B27 misfolding and spondyloarthropathies. Prion 2009;3:15-26.

19 Liu C-H, Raj S, Chen C-H, et al. HLA-B27-mediated activation of TNAP phosphatase promotes pathogenic syndesmophyte formation in ankylosing spondylitis. J Clin Invest 2019;129:5357-73.

20 Xueyi L, Lina C, Zhenbiao W, et al. Levels of circulating Th17 cells and regulatory $T$ cells in ankylosing spondylitis patients with an inadequate response to anti-TNF- $\alpha$ therapy. J Clin Immunol 2013;33:151-61.

21 Shen H, Goodall JC, Hill Gaston JS. Frequency and phenotype of peripheral blood Th17 cells in ankylosing spondylitis and rheumatoid arthritis. Arthritis Rheum 2009;60:1647-56.

22 Wang X, Lin Z, Wei Q, et al. Expression of IL-23 and IL-17 and effect of IL-23 on IL-17 production in ankylosing spondylitis. Rheumatol Int 2009:29:1343-7.

23 Wendling D. IL-23 and IL-17 in ankylosing spondylitis. Rheumatol Int 2010;30:1547.

24 Prevosto C, Goodall JC, Hill Gaston JS. Cytokine secretion by pathogen recognition receptor-stimulated dendritic cells in rheumatoid arthritis and ankylosing spondylitis. J Rheumatol 2012;39:1918-28.

25 Kehl AS, Corr M, Weisman MH. Review: enthesitis: new insights into pathogenesis, diagnostic modalities, and treatment. Arthritis Rheumatol 2016;68:312-22.

26 Lubberts E, Joosten LA, Oppers B, et al. IL-1-independent role of IL-17 in synovial inflammation and joint destruction during collageninduced arthritis. J Immunol 2001;167:1004-13.

27 Sherlock JP, Joyce-Shaikh B, Turner SP, et al. IL-23 induces spondyloarthropathy by acting on ROR- $\gamma t+C D 3+C D 4-C D 8$ entheseal resident T cells. Nat Med 2012;18:1069-76.

28 El-Zayadi AA, Jones EA, Churchman SM, et al. Interleukin-22 drives the proliferation, migration and osteogenic differentiation of mesenchymal stem cells: a novel cytokine that could contribute to new bone formation in spondyloarthropathies. Rheumatology 2017;56:488-93.

29 Wang Z, Tan J, Lei L, et al. The positive effects of secreting cytokines IL-17 and IFN- $\gamma$ on the early-stage differentiation and negative effects on the calcification of primary osteoblasts in vitro. Int Immunopharmacol 2018;57:1-10.

30 Goswami J, Hernández-Santos N, Zuniga LA, et al. A boneprotective role for IL-17 receptor signaling in ovariectomy-induced bone loss. Eur J Immunol 2009;39:2831-9.

31 Uluçkan Özge, Jimenez M, Karbach S, et al. Chronic skin inflammation leads to bone loss by IL-17-mediated inhibition of Wnt signaling in osteoblasts. Sci Trans/ Med 2016;8:330ra37.

32 Urist MR. Bone: formation by autoinduction. Science 1965:150:893-9.

33 Lories RJU, Derese I, Luyten FP. Modulation of bone morphogenetic protein signaling inhibits the onset and progression of ankylosing enthesitis. J Clin Invest 2005;115:1571-9.

34 Appel H, Ruiz-Heiland G, Listing J, et al. Altered skeletal expression of sclerostin and its link to radiographic progression in ankylosing spondylitis. Arthritis Rheum 2009;60:3257-62.

35 Heiland GR, Appel H, Poddubnyy D, et al. High level of functional Dickkopf-1 predicts protection from syndesmophyte formation in patients with ankylosing spondylitis. Ann Rheum Dis 2012;71:572-4.

36 Diarra D, Stolina M, Polzer K, et al. Dickkopf-1 is a master regulator of joint remodeling. Nat Med 2007;13:156-63.

37 Corr M. Wnt signaling in ankylosing spondylitis. Clin Rheumatol 2014;33:759-62.

38 Xie W, Zhou L, Li S, et al. Wnt//-catenin signaling plays a key role in the development of spondyloarthritis. Ann N Y Acad Sci 2016;1364:25-31. 
39 Lories RJ, Haroon N. Bone formation in axial spondyloarthritis. Best Pract Res Clin Rheumatol 2014;28:765-77.

40 Chen H-A, Chen C-H, Lin Y-J, et al. Association of bone morphogenetic proteins with spinal fusion in ankylosing spondylitis. J Rheumatol 2010;37:2126-32.

41 Chen M, Hu X, Wu M, et al. Serum Levels of OPG, RANKL, and RANKL/OPG Ratio in Patients with Ankylosing Spondylitis: A Systematic Review and Meta-analysis. Immunol Invest 2019;48:490-504.

42 Yang J, Xu S, Chen M, et al. Serum sclerostin and bone morphogenetic protein-2 levels in patients with ankylosing spondylitis: a meta-analysis. Calcif Tissue Int 2019;105:37-50.

43 Gracey E, Burssens A, Cambré I, et al. Tendon and ligament mechanical loading in the pathogenesis of inflammatory arthritis. Nat Rev Rheumatol 2020;16:193-207.

44 Lories RJU, Luyten FP, de Vlam K. Progress in spondylarthritis. mechanisms of new bone formation in spondyloarthritis. Arthritis Res Ther 2009;11:221.

45 Cambré I, Gaublomme D, Schryvers N, et al. Running promotes chronicity of arthritis by local modulation of complement activators and impairing T regulatory feedback loops. Ann Rheum Dis 2019;78:787-95.

46 Cambré I, Gaublomme D, Burssens A, et al. Mechanical strain determines the site-specific localization of inflammation and tissue damage in arthritis. Nat Commun 2018;9:4613.

47 Lubrano E, Perrotta FM. Secukinumab for ankylosing spondylitis and psoriatic arthritis. Ther Clin Risk Manag 2016;12:1587-92.

48 Braun J, Baraliakos X, Deodhar A, et al. Secukinumab shows sustained efficacy and low structural progression in ankylosing spondylitis: 4-year results from the MEASURE 1 study. Rheumatology 2019;58:859-68.

49 van der Heijde D, Baraliakos X, Hermann K-GA, et al. Limited radiographic progression and sustained reductions in MRI inflammation in patients with axial spondyloarthritis: 4-year imaging outcomes from the RAPID-axSpA phase III randomised trial. Ann Rheum Dis 2018;77:699-705.

50 Van Mechelen M, Lories R. Spondyloarthritis on the move: biomechanical benefits or harm. Curr Rheumatol Rep 2020;22:35.

51 Regel A, Sepriano A, Baraliakos X, et al. Efficacy and safety of nonpharmacological and non-biological pharmacological treatment: a systematic literature review Informing the 2016 update of the ASAS/EULAR recommendations for the management of axial spondyloarthritis. RMD Open 2017;3:e000397.

52 Zão A, Cantista P. The role of land and aquatic exercise in ankylosing spondylitis: a systematic review. Rheumatol Int 2017;37:1979-90.

53 Hilberdink B, Giesen F, Vliet Vlieland T, et al. Supervised group exercise in axial spondyloarthritis: patients' satisfaction and perspective on evidence-based enhancements. Arthritis Care Res 2020;72:829-37.

54 O'Dwyer T, O'Shea F, Wilson F. Exercise therapy for spondyloarthritis: a systematic review. Rheumatol Int 2014;34:887-902.
55 Redeker I, Hoffmann F, Callhoff J, et al. Determinants of psychological well-being in axial spondyloarthritis: an analysis based on linked claims and patient-reported survey data. Ann Rheum Dis 2018;77:1017-24.

56 Millner JR, Barron JS, Beinke KM, et al. Exercise for ankylosing spondylitis: an evidence-based consensus statement. Semin Arthritis Rheum 2016;45:411-27.

57 Lubrano E, D'Angelo S, Parsons WJ, et al. Effects of a combination treatment of an intensive rehabilitation program and etanercept in patients with ankylosing spondylitis: a pilot study. J Rheumatol 2006;33:2029-34.

58 Lubrano E, Spadaro A, Amato G, et al. Tumour necrosis factor alpha inhibitor therapy and rehabilitation for the treatment of ankylosing spondylitis: a systematic review. Semin Arthritis Rheum 2015;44:542-50.

59 van der Heijde D, Ramiro S, Landewé R, et al. 2016 update of the ASAS-EULAR management recommendations for axial spondyloarthritis. Ann Rheum Dis 2017;76:978-91.

60 Wervers K, Herrings I, Luime JJ, et al. Association of physical activity and medication with Enthesitis on ultrasound in psoriatic arthritis. $J$ Rheumatol 2019;46:1290-4.

61 Debusschere K, Cambré I, Gracey E, et al. Born to run: the paradox of biomechanical force in spondyloarthritis from an evolutionary perspective. Best Pract Res Clin Rheumatol 2017;31:887-94.

62 Ramiro S, Landewé R, van Tubergen A, et al. Lifestyle factors may modify the effect of disease activity on radiographic progression in patients with ankylosing spondylitis: a longitudinal analysis. $R M D$ Open 2015;1:e000153.

63 Amrein K, Amrein S, Drexler C, et al. Sclerostin and its association with physical activity, age, gender, body composition, and bone mineral content in healthy adults. J Clin Endocrinol Metab 2012;97:148-54.

64 Deymier AC, Schwartz AG, Cai Z, et al. The multiscale structural and mechanical effects of mouse supraspinatus muscle unloading on the mature enthesis. Acta Biomater 2019;83:302-13

65 Jennings F, Lambert E, Fredericson M. Rheumatic diseases presenting as sports-related injuries. Sports Med 2008;38:917-30.

66 Sveaas SH, Dagfinrud H, Berg IJ, et al. High-Intensity exercise improves fatigue, sleep, and mood in patients with axial spondyloarthritis: secondary analysis of a randomized controlled trial. Phys Ther 2020;100:1323-32.

67 Sveaas SH, Bilberg A, Berg IJ, et al. High intensity exercise for 3 months reduces disease activity in axial spondyloarthritis (axSpA): a multicentre randomised trial of 100 patients. Br J Sports Med 2020;54:292-7.

68 Kim HR, Hong YS, Park S-H, et al. Low bone mineral density predicts the formation of new syndesmophytes in patients with axial spondyloarthritis. Arthritis Res Ther 2018;20:231.

69 Strope MA, Nigh P, Carter MI, et al. Physical activity-associated bone loading during adolescence and young adulthood is positively associated with adult bone mineral density in men. Am J Mens Health 2015;9:442-50. 